\title{
Human Amniotic Epithelial Cells Affect the Functions of Neutrophils
}

\author{
Razieh Alipour ${ }^{1}$, Hossein Motedayyen ${ }^{2}$, Nasrin Sereshki ${ }^{3}$, \\ Mitra Rafiee ${ }^{4}$, Fereshteh Alsahebfosul ${ }^{1}$, Abbasali Pourazar ${ }^{1}$ \\ ${ }^{1}$ Department of Immunology, Medical School, Isfahan University of Medical Sciences, Isfahan, Iran \\ ${ }^{2}$ Department of Immunology, Autoimmune Diseases Research Center, Kashan University of Medical Sciences, Kashan, Iran \\ ${ }^{3}$ Department of Immunology, Asadabad School of Medical Science, Asadabad, Iran \\ ${ }^{4}$ Cellular and Molecular Research Center, Department of Immunology, Medical School, \\ Birjand University of Medical Sciences, Birjand, Iran
}

Background and Objectives: As a stem cell group, Human amniotic epithelial cells (HAECs) have numerous advantages over their embryonic and adult counterparts for therapeutic utility. They are closer to clinical applications compared to other stem cell types. Additionally, the anti-inflammatory and immunoregulatory properties of HAECs toward several immune cells have been shown previously. Nevertheless, despite the ever-increasing importance of neutrophils in the immune and non-immune processes, a few studies investigated the interaction of neutrophils and HAECs. To increase the current knowledge of HAECs immunology which is necessary for optimizing their future clinical applications, here we explored the effect of HAECs on two chief neutrophil functions; respiratory burst and phagocytosis.

Methods and Results: Freshly isolated human blood neutrophils were co-cultured with different number of HAECs for about 24 or 48 hours, then the oxidative burst and phagocytosis of stimulated neutrophils were assessed and compared. The results demonstrated a substantial elevation in the phagocytosis percentage, conversely a significant reduction in the oxidative burst of HAECs-cocultured neutrophils. These effects were dose-dependent, but did not show similar patterns. Likewise, the elongation of coculture period inversely influenced the HAECs-induced effects on the two neutrophil functions.

Conclusions: The present study, for the first time, investigated the HAECs-mediated effects on the two main neutrophil functions. The findings suggest that HAECs by enhancement of phagocytic ability and simultaneously, attenuation of oxidative burst capacity of neutrophils protect the fetus from both microbial treats and oxidative stress and their consequent inflammation; thus corroborate the current anti-inflammatory vision of HAECs.

Keywords: Human amniotic epithelial cells, Neutrophils, Oxidative burst, Phagocytosis

Received: December 18, 2019, Revised: February 10, 2020, Accepted: February 10, 2020, Published online: April 30, 2020 Correspondence to Abbasali Pourazar

Department of Immunology, Medical School, Isfahan University of Medical Sciences, Hezar Jerib Street, Isfahan 81746-73695, Iran Tel: +98-31-379229031, Fax: +98-36688597

E-mail: pourazar@med.mui.ac.ir

(c) This is an open-access article distributed under the terms of the Creative Commons Attribution Non-Commercial License (http://creativecommons.org/ licenses/by-nc/4.0/), which permits unrestricted non-commercial use, distribution, and reproduction in any medium, provided the original work is properly cited.

Copyright (c) 2020 by the Korean Society for Stem Cell Research

\section{Introduction}

Nowadays, various types of stem cells have become promising tools to treat a variety of human disorders. Human amniotic epithelial cells (HAECs), among other stem cells, have attracted a lot interest in the therapeutic fields. HAECs are derived from the amniotic layer of the placenta which is a biological waste that discarded at birth. Thus, compared to most adult stem cells (ASCs) (e.g. from blood, bone marrow, adipose tissue or teeth), 
HAECs are available with noninvasive methods and reduced moral obstacles. Moreover, HAECs can be obtained in a larger number and comprise a younger population with higher differentiation potential in respect to most other ASCs (1-3).

The chief advantages of HAECs over embryonic stem cells (ESCs) are the lack of tumorigenicity as well as the ease of accessibility without ethical problems (1).

The special immunological properties of HAECs, besides the above mentioned features, also make the use of them in clinical settings more rational. HAECs do not express histocompatibility antigens (HLA-A, B or DR) and costimulatory molecules but express immunoregulatory molecules like HLA-G and CD95, so they are poorly immunogenic (4). Additionally, HAECs have anti-inflammatory properties, they possess inhibitory capacity toward activated inflammatory cells and directed the ongoing immune responses to a more immunoregulatory and tolerogenic condition (5).

A lot of scientists focused on lymphocytes, especially $\mathrm{T}$ cells, and demonstrated that HAECs can hinder their cytokine production and proliferation (6). Some researches, also have considered the innate immune cells like as macrophages, monocytes and dendritic cells and reinforced that HAECs can skew the immune system to an anti-inflammatory phenotype (7). However, a few reports have investigated the interaction of neutrophils and HAECs. Indeed, except limited reports on the migration and apoptosis (8-10), we could not find scientific data on other functions of HAECs-affected neutrophils such as phagocytosis, oxidative burst, chemotaxis or NET (neutrophil extracellular trap) formation. Reviewing the scientific literature, it appears that the effect of HAECs on neutrophils is the least-studied immunological aspect of HAECs.

Interestingly, neutrophils, the majority of polymorphonuclear (PMN) leukocytes, form an important part of the innate immunity. In the conventional view, they are the most abundant leukocytes that quickly reach the infection site and their long-known function is phagocytosis (11). Recent studies, however, have revealed that neutrophils are much more important cells than we traditionally thought. In addition to the killing of microorganisms, they have key roles in tissue homeostasis, inducing and resolving the inflammation as well as regulation of the immune system and angiogenesis. Also, their aberrant functionality is involved in the pathobiology of many human diseases (12). Overall, based on the current findings, neutrophils are versatile cells which actively participate in many of physiologic and pathologic processes (13).
Given the newfound key roles of neutrophils in various biological processes, further understanding of how HAECs interact with neutrophils is crucial to design and develop successful therapeutic modalities using HAECs. Hence, in the present study, we investigated the effect of HAECs on the two important functions of neutrophils; oxidative burst and phagocytosis to increase the current understanding of HAECs immunology, in order to improve the clinical utility of them. To this end, freshly isolated human neutrophils were co-cultured with different numbers of HAECs for about 24 or 48 hours, then the oxidative burst and phagocytosis of stimulated neutrophils were assessed and compared.

\section{Materials and Methods}

\section{Blood collection}

About $20 \mathrm{ml}$ venous blood was collected from healthy volunteers into polypropylene tubes contained EDTA-Acid Citrate Dextrose (ACD) $(10 \%[\mathrm{w} / \mathrm{v}] \sim 15 \%[\mathrm{v} / \mathrm{v}])$.

The study was approved by the Ethics Committee of the Isfahan University of Medical Sciences and performed in accordance with the Declaration of Helsinki. All participates were obtained with written informed consent.

\section{Human neutrophil isolation}

After removing platelet-rich-plasma (PRP) by centrifugation $\left(250 \mathrm{~g}, 18^{\circ} \mathrm{C}, 15 \mathrm{~min}\right)$, and then red blood cells (RBCs) sedimentation by 6\% dextran (Sigma-Aldrich), the obtained leukocyte rich suspension decanted onto a 2-layered discontinuous density gradient of Percoll (86\% and 55\% isotonic Percoll [Santa Cruz]), and was spun (480 g, $18^{\circ} \mathrm{C}, 17 \mathrm{~min}$, brake off) to separate the mononuclear cells and granulocytes in two distinct layers. To prevent the mixing, the mononuclear cells layer was firstly aspirated, then the neutrophils layer was carefully removed and cells were washed by RPMI 1640 (Gibco) (5 min, $300 \mathrm{~g}, 18^{\circ} \mathrm{C}$ ). Cell viability and count were evaluated by the Trypan blue exclusion method and neutrophils were suspended gently in RPMI medium at known concentration.

\section{Preparation of HAECs}

The vials of frozen HAECs were thawed according standard protocol (14) and were cultured in $25-\mathrm{cm}^{2}$ tissue culture flasks in high glucose Dulbecco's modified Eagle's medium (high glucose DMEM; Gibco) supplemented with 15\% FCS (Sigma-Aldrich) and 1\% penicillin/streptomycin (Sigma-Aldrich, USA). These cells have been already isolated from full-term human placenta obtained from healthy women (15). Approximately, after $24 \mathrm{~h}$ of in- 
cubation $\left(5 \% \mathrm{CO} 2,37^{\circ} \mathrm{C}, 90 \%\right.$ humidity) the adherent cells were trypsinized and plated at diminishing numbers on 24 -well plates $\left(4 \times 10^{5}, 2 \times 10^{5}, 8 \times 10^{4}\right.$ and $4 \times 10^{4}$ cell/well $)$ for further coculture with neutrophils. Before the adding neutrophils, HAECs were allowed to adhere to the plate for 16 to $18 \mathrm{~h}$.

\section{Cocultures}

In this experiment, the same number of human granulocytes were cultured alone or co-cultured with HAECs at different ratios $(1: 1,1: 2,1: 5$ and $1: 10)$. For this, the medium of HAECs cultures (in 24-well plates) were aspired and $4 \times 10^{5}$ freshly isolated human PMNs in $1 \mathrm{ml}$ of RPMI, completed by $10 \%$ FCS and $1 \%$ penicillin/ streptomycin, were added to each well. The cocultures incubated for $24 \mathrm{~h}$ and $48 \mathrm{~h}$ at $37^{\circ} \mathrm{C}$ in a humidified atmosphere of $5 \% \mathrm{CO}$. All assays were performed in duplicate.

\section{Measurement of oxidative burst}

Once the designated incubations were complete, neutrophils were harvested by aspirating the medium, washed and resuspended in RPMI without serum, then divided into two aliquots for further phagocytosis and respiratory burst study. The measurement of neutrophil respiratory burst (the release of reactive oxygen species [ROS]) was done using oxidation of dihydrorhodamine-123 (DHR) (Santa Cruz). The neutrophils suspension was activated by Cell Activation Cocktail (Biolegend) for $20 \mathrm{~min}$ at $37^{\circ} \mathrm{C}$, $5 \% \mathrm{CO} 2,90 \%$ humidity. Then, DHR at final concentration of $1 \mu \mathrm{M}$ was added and the cells were re-incubated for another $20 \mathrm{~min}\left(37^{\circ} \mathrm{C}, 5 \% \mathrm{CO} 2,90 \%\right.$ humidity). Afterwards, the cells were placed into an ice bath to stop reaction $(10 \mathrm{~min})$. After washing once with cold PBS (300 g, $3 \mathrm{~min}, 18^{\circ} \mathrm{C}$ ), cells were resuspended in FACS solution (flow cytometry sheath fluid) contained $0.5 \%$ formaldehyde and analyzed by the flow cytometry. Negative controls were also treated in the same way but without activation.

\section{Phagocytosis assay}

To determine the function of HAECs-cocultured neutrophils, the amount of the phagocytosis of them after activation was assessed; briefly, an aliquot of the washed neutrophils (after coculture) were stimulated with 50 $\mathrm{ng} / \mathrm{ml}$ endotoxin (LPS from Escherichia coli, serotype 0111: B4, Sigma) at $37^{\circ} \mathrm{C}, 5 \% \mathrm{CO} 2,90 \%$ humidity for 30 min in 96-well v-bottom plate. Afterwards, fluorescent latex beads $(1.0 \mu \mathrm{m}$ mean size, Sigma) were added (the final concentration of beads were about $2 \times 10^{5}$ beads $/ \mathrm{ml}$ ) and neutrophils were incubated for another $30 \mathrm{~min}\left(37^{\circ} \mathrm{C}\right.$,
5\% CO2, 90\% humidity). After incubation, the cells were washed twice with a large volume of cold PBS and resuspended in cold FACS solution contained $0.5 \%$ formaldehyde, then were run on the flow cytometer. Negative controls were treated as mentioned except LPS stimulation.

Flow cytometry was performed using a FACSCalibur flow cytometer (Becton Dickinson). The data were acquired using CellQuest (Becton Dickson) and then analyzed by FlowJo software version $\mathrm{X}$.

\section{Statistical analysis}

Statistics were calculated using IBM SPSS Statistics software (version 25). Results are expressed as mean \pm standard error of the mean (SEM). Tests of ANOVA or repeated measurements ANOVA (indicated in the figures legends) were used to statistically evaluate the difference between groups, as appropriate. In all cases, p-values lower than 0.05 were considered as statistically significant.

\section{Results}

\section{HAECs inversely affected the phagocytosis and oxidative burst of neutrophils}

In the phagocytosis assessment, the percentage of fluorescent neutrophils (which phagocytosed the beads) were shown as phagocytosis percentage (Fig. 1). For the oxidative burst experiments, the results were expressed as an oxidative burst index (OBI); that is, the ratio of mean fluorescence of stimulated neutrophils to mean fluorescence of unstimulated neutrophils (Fig. 2A).

As a first approach, we compared the neutrophils cultured alone vs those cocultured with HAECs and found that HAECs reduced the level of oxidative burst significantly (Fig. 3A). In contrast, HAECs increased markedly the amount of phagocytosis (Fig. 3B).

\section{The number of HAECs as well as the duration of coculture differently affected the interaction between neutrophils and hAECs}

Then we inspected the effects of coculture duration (time) and HAECs number (ratios) as well as their interaction on the two functions of granulocytes.

In cell-ratio effect analysis, HAECs significantly augmented the phagocytosis in all ratios $(1: 1,1: 2,1: 5$, and $1: 10) ;(p=0.012)$ and at the $1: 10$ ratio (of HAECs to neutrophils), the highest level of phagocytosis was seen (Fig. 2B and 4B). In the case of respiratory burst, all four cocultures showed a reduced level of OBI that was statistically significant except at $1: 1$ ratio (hAECs : neutrophils) $(p=0.00)$. The strongest inhibition was seen at 


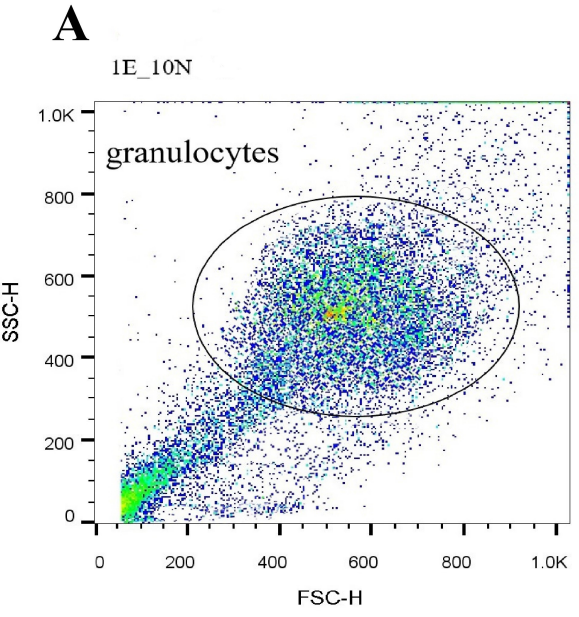

B

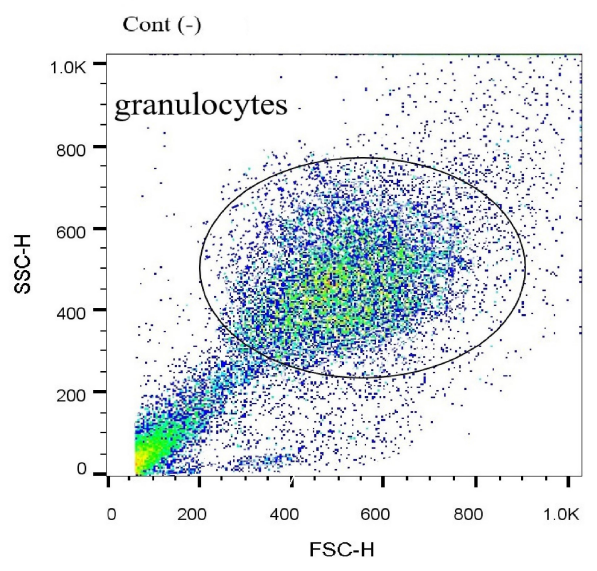

Fig. 1. HAECs influenced the phagocytosis of human blood granulocytes in cocultures. Isolated blood granulocytes were cocultured with hAECs at various ratio and time (as detailed in the text). After coculture, neutrophils and HAECs were separated by adherence. For phagocytosis assay, the neutrophils were stimulated and added by fluorescent 1.0 $\mu \mathrm{m}$ diameter beads and run on the flow cytometer. The uptake of fluorescent beads causes the neutrophils become fluorescent too; thus the percent of fluorescent granulocytes presented as phagocytosis percentage (A); unstimulated neutrophils were used as negative control (B).
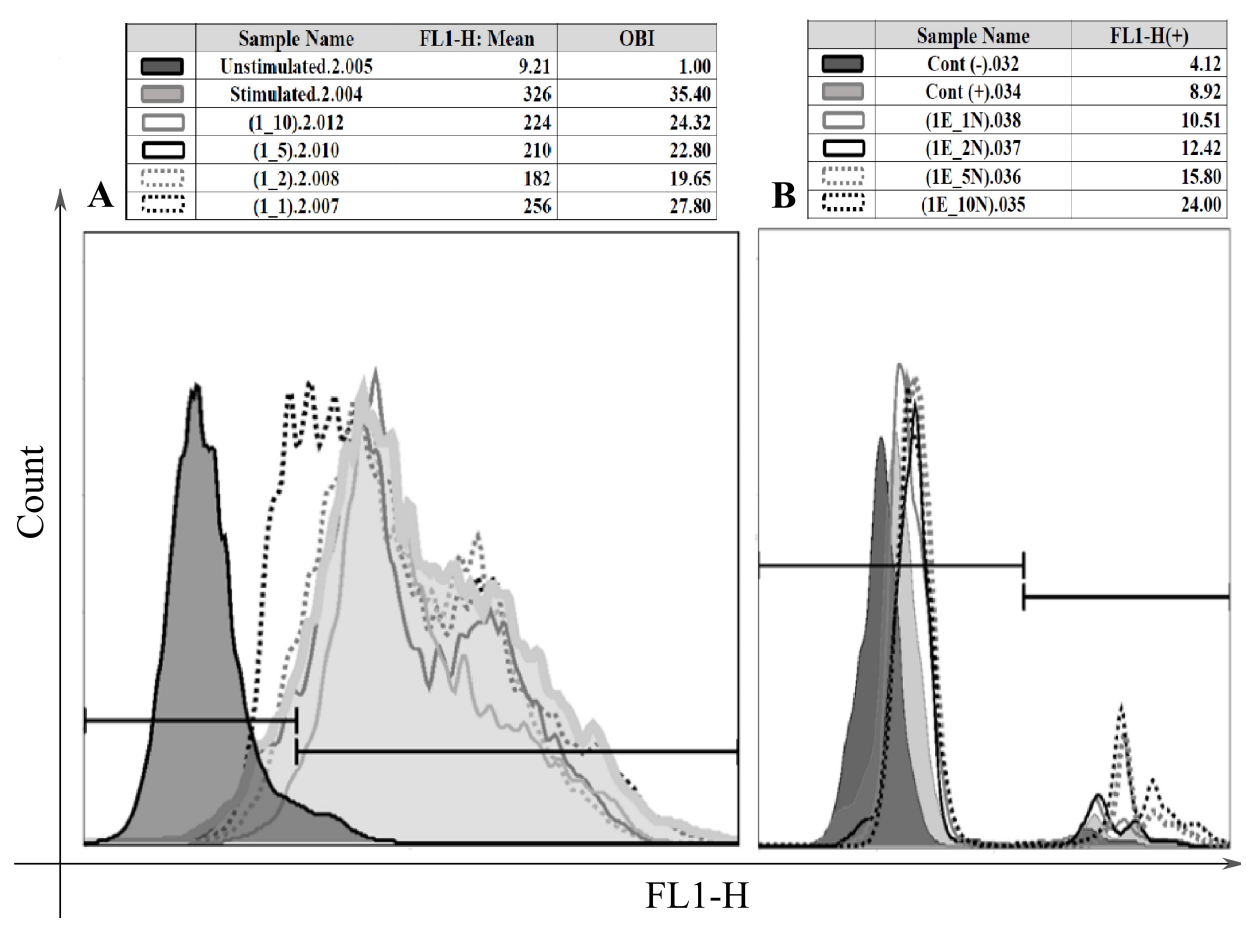

Fig. 2. The effects of HAECs number on the functions of granulocytes. The oxidative burst capacity of human granulocytes changed when they were cocultured with different number of HAECs. The levels of respiratory burst were assessed by flow cytometry and for comparison of the results, $\mathrm{OBI}$ (oxidative burst index) was calculated; that is the ratio of mean fluorescence of stimulated neutrophils to mean fluorescence of unstimulated neutrophils (A). The coculture of human granulocytes with various number of hAECs altered the phagocytosis percentage of the neutrophils (B). 
$1: 2$ ratio which had the lowest level of OBI. Although not significant, there was a tendency for higher OBI from $1: 2$ to $1: 10$ ratios (Fig. $2 \mathrm{~A}$ and $4 \mathrm{~A}$ ).

Regarding the coculture duration, the amounts of the oxidative burst, as well as phagocytosis, were elevated significantly in $48 \mathrm{~h}$ - cocultures when compared to $24 \mathrm{~h}$ cocultures (for OBI: $p=0.034$; and for phagocytosis percentage: $p=0.00$ ). This means that the inhibitory effect of HAECs on oxidative burst diminished, but the stimulatory effect of HAECs on phagocytosis increased by the time (Fig. 4).

Considering the statistical interplay between the two variables, there was no interaction between time and cell ratio for oxidative burst, as the overall trends of OBI change over the ratios did not significantly differ between the two individual periods of co-culture ( $24 \mathrm{~h}$ and $48 \mathrm{~h}$ ) (Fig. 4A). Conversely, the patterns of changes of phagocytosis over the ratios were altered statistically significant between the two co-culture's periods, indicating the existence of an interaction between the two factors (i.g. time and ratio) (Fig. 4B).

\section{Discussion}

Human amniotic epithelial cells (HAECs), isolated from human amniotic membrane (AM) of term placenta, possess all criteria of stemness. Due to many superiorities over other stem cells, HAECs have been viewed as more
A

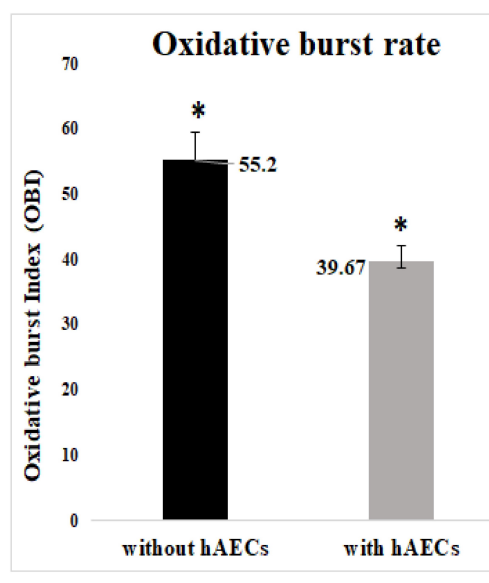

B

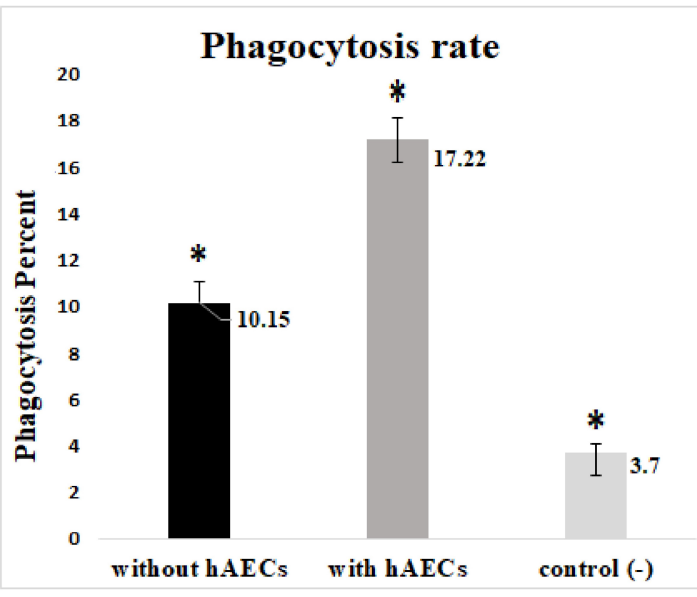

Fig. 3. The impact of HAECs on the oxidative burst and phagocytosis of granulocytes. On the whole, the data analysis revealed that co-cultivation of neutrophils with HAECs reduced the level of oxidative burst $(p=0.000) \quad(A)$; but enhance the phagocytosis $(p=0.000)$ in comparison to neutrophils cultured alone (without hAECs) (one way ANOVA, $\mathrm{n}=5)(\mathrm{B})$; hAECs: human amniotic epithelial cells, control $(-)$ : resting (unstimulated) neutrophils.
A

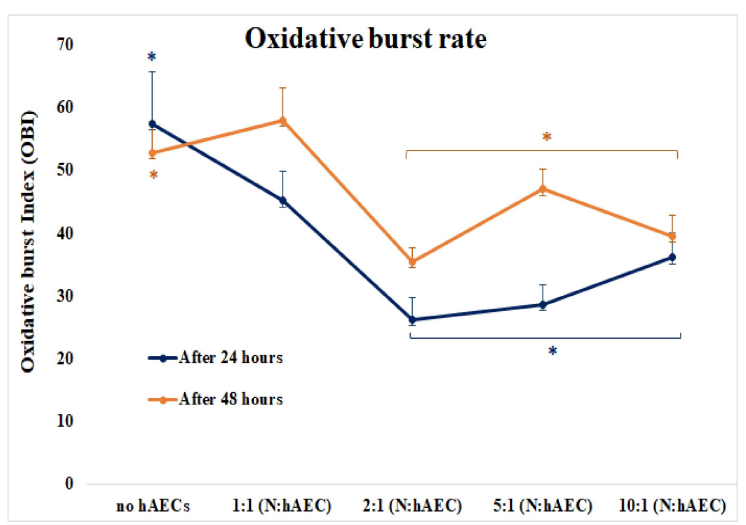

B

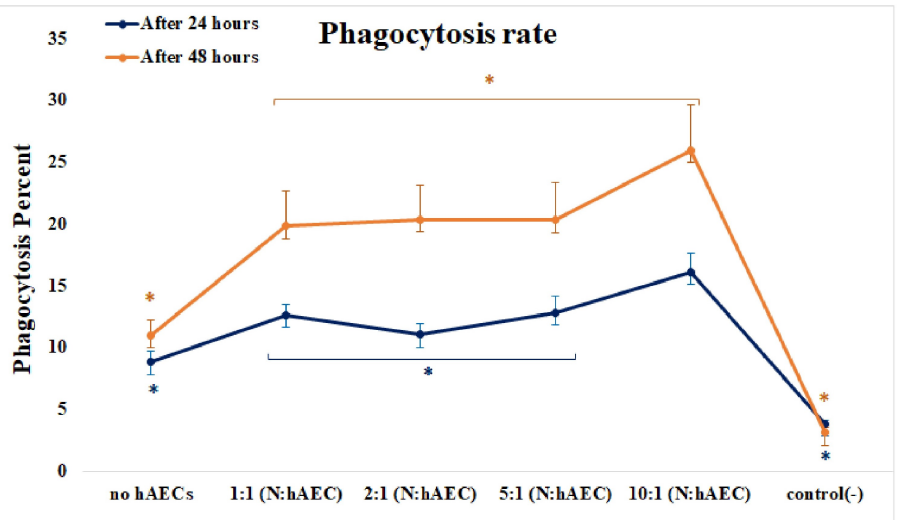

Fig. 4. HAECs affected neutrophil functions time- and dose-dependently. The HAECs-induced inhibition of respiratory burst of neutrophils differed in the presence of different number of HAECs and diminished by time. However, the ratio groups do not differ statistically significant in their degree of change in the OBI over time (Repeated measurement ANOVA, $n=5, p=0.104$ ) (A). The phagocytic activity of neutrophils were raised considerably in all ratios of HAECs to neutrophils, and the lowest ratio caused the highest level; it also showed an increase by time. Additionally, there was an interaction between time and cell ratio; the effect of cell numbers on the phagocytosis varied over time (Repeated measurement ANOVA, $n=5, p=0.034$ ) (B). 
attractive candidates for many therapeutic settings. Additionally, compared to many of their counterparts, HAECs are closer to clinical applications, because AM has been using in several medical fields including ocular surgery and burn or wound healing for more than one hundred years $(1,16)$.

Like other stem cells, special immunoregulatory properties have been known for HAECs; so far, numerous studies have investigated the influence of HAECs on phenotype, activation, and functions of various leukocytes including $\mathrm{T}$ cells and their subsets, B cells, NK cells, monocytes, and dendritic cells $(6,7)$.

Despite the ever-increasing importance of neutrophils as immune cells and also as cells which participate in many physiological and pathological processes (12), the studies which explored the effect of HAECs on neutrophils are infrequent (8-10). In the present study, therefore, the modulation of two important neutrophil functions, namely respiratory burst and phagocytosis, by HAECs have been addressed in vitro. As past studies have shown that the effect of HAECs are time and dose-dependent (17-19), here we examined the effect of different number of HAECs on the neutrophil functions at different times; after 24 and 48 hours of coculturing.

Our results demonstrate a strong inhibition of HAECs on the oxidative burst of isolated blood granulocytes. Unlike to the respiratory burst, HAECs potentiated the phagocytic ability of neutrophils dramatically. We could not find any other research focusing on these neutrophil activities in the presence of HAECs. But these findings are in agreement with the previous results implying the increasing of phagocytic activity and or decreasing the production of ROS by both activated and resting PMNs upon contact with various types of human mesenchymal stem cells (H-MSCs) or their conditioned media (20-23). HAECs are common in many immunoregulatory properties with H-MSCs $(6,17)$ and it is not unexpected that they affect neutrophils in a similar way to H-MSCs.

We also found the inhibition of oxidative burst as well as the enhancement of phagocytosis are time dependent as the inhibition was impaired after prolongation of coculture time from $24 \mathrm{~h}$ to $48 \mathrm{~h}$, but by extension of period of contact to HAECs, neutrophil phagocytosis further augmented meaningfully. No human study compared the respiratory burst and phagocytic activity of neutrophils in the presence of stem cells during the time. Indeed, one of the limitations of the current work is that the changes in the two functions of human neutrophils upon contact with HAECs and other stem cells, particularly H-MSCs, under similar conditions were not compared and its pro- viding data were cofounded to HAECs only. However, analogous to our report, in studies on rats, the increased levels of oxidative burst and or the decreased levels of phagocytic ability of neutrophils were observed after $48 \mathrm{~h}$ of coculturing with MSCs, compared to $24 \mathrm{~h}$ cocultures (24, 25).

The dose-dependency of the immunoregulatory capacity of HAECs on different immune cells have been highlighted before (17-19); it seems that HAECs exert their effect on phagocytosis and oxidative burst of neutrophils in a dose-dependent manner too. But none of neutrophil functions evaluated here replicate exactly the results of past researches in which usually progressive dilution of HAECs numbers are accompanied with less pronounced inhibition on leukocytes (17-19). As it is seen (in the Results section), when HAEC : neutrophil ratio was highest $(1: 1)$, the level of respiratory burst did not differ significantly. Instead, the highest inhibition was at the ratio of $1: 2$ and by decreasing the ratio the inhibition decreased too but not significantly. In the case of phagocytosis, of note, by decreasing the ratio of HAECs : neutrophils, the phagocytic activity increased, as we observed the lowest and highest level of phagocytosis, respectively at the highest and lowest ratio of HAECs to neutrophils. These incompatible findings may be due to the fact that HAECs differentially regulate different populations of immune cells as it has been presented recently $(26,27)$.

The present dominant view of HAECs is that they are immunomodulatory cells which express or secrete surface/soluble immunoreactive molecules $(10,27,28)$. Some of these molecules such as HLA-G (29), PGE2 (30, 31), IL-10 (32), and IDO (33) have already been shown that can influence neutrophils as well. Therefore, HAECs are expected to modify neutrophil activities. However, the obtained results showed that the effect of HAECs on neutrophils was not exclusively suppressive. We observed that HAECs significantly enhanced the phagocytic ability of cocultured neutrophils and decreased the production of ROS from PMA-stimulated neutrophils. Although these results need to be verified by future studies on HAECs, reviewing the physiological mission of AM help explaining the results.

One of the important roles of the placenta is to protect the embryo from various threats, namely traumas, oxidative stress, microbial infections and also maternal immune responses to the semi-allogenic fetus $(16,34,35)$. Since the anatomical lactation, AM and therefore HAECs have a specific role in the protection. These cells are in contact to amniotic fluid in which the fetus is floating $(16,36)$. The amniotic fluid harbor a little population of immune 
cells including neutrophils (37). Thus the interaction of HAECs and immune cells should be regulated stringently to inhibit the unnecessary immune processes (such as anti-fetus maternal immunity) and prevent the uncontrolled inflammation that can endamage the fetus. At the same time, HAECs should preserve the anti-microbial immune responses, otherwise, the fetus may be injured.

Regarding neutrophils, the oxidative burst is one of their cytotoxic mechanisms to destroy invader microbes, but the improper or excessive production of ROS can result in oxidative stress and subsequently inflammation (38). On the other hand, neutrophils phagocytosis is an effective defense mechanism that eliminates pathogens from the fetus milieu (39); protects the fetus from their pathogenicity and also prevents the prolongation of inflammation.

Overall, we can speculate that HAECs act simultaneously through strengthening the phagocytic ability and attenuating the oxidative burst capacity of neutrophils to provide the best fetus protection from both microbial threats and potential oxidative stress and their consequent inflammation. This speculation is supported by the observation that the phagocytosis was increased by the reduction of the number of HAECs in cocultures and by increasing the incubation time. As far as the studies found out, neutrophils exist in amniotic fluid in low number (37), it means that the natural ratio of HAECs to neutrophils is high in normal condition. When an intra-amniotic infection happens and the subsequent phagocytic activity is needed, the increased number of neutrophils in amniotic fluid occurs $(37,40)$ and consequently the ratio of HAECs to neutrophils reduces, this may induce HAECs to potentiate phagocytic ability of neutrophils more and help to resolve the infection.

To our knowledge, this is the first study investigated the HAECs-mediated effects on the two main neutrophil functions; phagocytosis and respiratory burst. HAECs differently influenced the phagocytic ability and respiratory burst capacity of neutrophils in a time- and dose-dependent manner. However, the dose- and time- dependent impacts of HAECs on the neutrophils seen here were not the same as reported previously for the HAECs-induced effects on other immune cells. The findings of the present study suggest that HAECs regulate neutrophil functions in a way to protect the fetus from microbial infections while prevent potentially harmful production of ROS, consequently dampen detrimental hyperactive inflammation in the fetus environment. The present results corroborate the current anti-inflammatory vision of HAECs.

\section{Acknowledgments}

The authors thank all individuals who participated in this study. This work was financially supported by Research and Technology Assistant of Isfahan University of Medical Sciences.

\section{Potential Conflict of Interest}

The authors have no conflicting financial interest.

\section{References}

1. de la Torre P, Pérez-Lorenzo MJ, Flores AI. Human placenta-derived mesenchymal stromal cells: a review from basic research to clinical applications. In: Valarmathi MT, editor. Stromal Cells. London: IntechOpen; 2019. 230-231

2. Miki T. A rational strategy for the use of amniotic epithelial stem cell therapy for liver diseases. Stem Cells Transl Med 2016;5:405-409

3. Ilancheran S, Michalska A, Peh G, Wallace EM, Pera M, Manuelpillai U. Stem cells derived from human fetal membranes display multilineage differentiation potential. Biol Reprod 2007;77:577-588

4. Miki T. Stem cell characteristics and the therapeutic potential of amniotic epithelial cells. Am J Reprod Immunol 2018;80:e13003

5. Motedayyen H, Fathi F, Fasihi-Ramandi M, Ali Taheri R. The effect of lipopolysaccharide on anti-inflammatory and pro-inflammatory cytokines production of human amniotic epithelial cells. Reprod Biol 2018;18:404-409

6. Magatti M, Cargnoni A, Silini A, Parolini O. Epithelial and mesenchymal stromal cells from the amniotic membrane. In: Atala A, Cetrulo KJ, Taghizadeh RR, Murphy SV, Cetrulo CL, editors. San Diego: Elsevier Science \& Technology; 2018. 147-155

7. Insausti CL, Blanquer $M$, García-Hernández AM, Castellanos G, Moraleda JM. Amniotic membrane-derived stem cells: immunomodulatory properties and potential clinical application. Stem Cells Cloning 2014;7:53-63

8. Li H, Niederkorn JY, Neelam S, Mayhew E, Word RA, McCulley JP, Alizadeh H. Immunosuppressive factors secreted by human amniotic epithelial cells. Invest Ophthalmol Vis Sci 2005;46:900-907

9. Zhou S, Chen J, Feng J. The effects of amniotic membrane on polymorphonuclear cells. Chin Med J (Engl) 2003;116: 788-790

10. Parolini O, Souza-Moreira L, O'Valle F, Magatti M, Hernandez-Cortes P, Gonzalez-Rey E, Delgado $M$. Therapeutic effect of human amniotic membrane-derived cells on experimental arthritis and other inflammatory disorders. Arthritis Rheumatol 2014;66:327-339

11. Kruger P, Saffarzadeh M, Weber AN, Rieber N, Radsak M, von Bernuth H, Benarafa C, Roos D, Skokowa J, Hartl D. Neutrophils: between host defence, immune modulation, and tissue injury. PLoS Pathog 2015;11:e1004651

12. Nicolás-Ávila JÁ, Adrover JM, Hidalgo A. Neutrophils in 
homeostasis, immunity, and cancer. Immunity 2017;46: $15-28$

13. Yang F, Feng C, Zhang X, Lu J, Zhao Y. The diverse biological functions of neutrophils, beyond the defense against infections. Inflammation 2017;40:311-323

14. Liu W, Chen G. Cryopreservation of human pluripotent stem cells in defined medium. Curr Protoc Stem Cell Biol 2014;31:1C.17.1-1C.17.13

15. Motedayyen H, Esmaeil N, Tajik N, Khadem F, Ghotloo S, Khani B, Rezaei A. Method and key points for isolation of human amniotic epithelial cells with high yield, viability and purity. BMC Res Notes 2017;10:552

16. Islam R, Rahman MS, Asaduzzaman SM, Rahman MS. Properties and therapeutic potential of human amniotic membrane. Asian J Dermatol 2015;7:1-12

17. Wolbank S, Peterbauer A, Fahrner M, Hennerbichler S, van Griensven M, Stadler G, Redl H, Gabriel C. Dose-dependent immunomodulatory effect of human stem cells from amniotic membrane: a comparison with human mesenchymal stem cells from adipose tissue. Tissue Eng 2007;13:1173-1183

18. Motedayyen H, Zarnani AH, Tajik N, Ghotloo S, Rezaei A. Immunomodulatory effects of human amniotic epithelial cells on naive $\mathrm{CD} 4^{+} \mathrm{T}$ cells from women with unexplained recurrent spontaneous abortion. Placenta 2018;71:31-40

19. Banas RA, Trumpower C, Bentlejewski C, Marshall V, Sing $\mathrm{G}$, Zeevi A. Immunogenicity and immunomodulatory effects of amnion-derived multipotent progenitor cells. Hum Immunol 2008;69:321-328

20. Raffaghello L, Bianchi G, Bertolotto M, Montecucco F, Busca A, Dallegri F, Ottonello L, Pistoia V. Human mesenchymal stem cells inhibit neutrophil apoptosis: a model for neutrophil preservation in the bone marrow niche. Stem Cells 2008;26:151-162

21. Chen CP, Chen YY, Huang JP, Wu YH. The effect of conditioned medium derived from human placental multipotent mesenchymal stromal cells on neutrophils: possible implications for placental infection. Mol Hum Reprod 2014;20:1117-1125

22. Salami F, Tavassoli A, Mehrzad J, Parham A. Immunomodulatory effects of mesenchymal stem cells on leukocytes with emphasis on neutrophils. Immunobiology 2018;223:786-791

23. Khan I, Zhang L, Mohammed M, Archer FE, Abukharmah J, Yuan Z, Rizvi SS, Melek MG, Rabson AB, Shi Y, Weinberger B, Vetrano AM. Effects of Wharton's jelly-derived mesenchymal stem cells on neonatal neutrophils. J Inflamm Res 2014;8:1-8

24. Pourtayeb S, Abtahi Froushani SM. Nicotine can modulate the effects of the mesenchymal stem cells on neutrophils. Adv Med Sci 2017;62:165-170

25. Galeh HEG, Delirezh N, Abtahi Froushani SM, Afzale Ahangaran N. Calcitriol modulates the effects of the supernatants of bone-marrow-derived mesenchymal stem cells on neutrophil functions. Turk J Biol 2014;38:365-370

26. Morandi F, Horenstein AL, Quarona V, Faini AC, Castella
B, Srinivasan RC, Strom SC, Malavasi F, Gramignoli R. Ectonucleotidase expression on human amnion epithelial cells: adenosinergic pathways and dichotomic effects on immune effector cell populations. J Immunol 2019;202: 724-735

27. Laranjeira $P$, Duque $M$, Vojtek $M$, Inácio $M J$, Silva I, Mamede AC, Laranjo M, Pedreiro S, Carvalho MJ, Moura P, Abrantes AM, Maia CJ, Domingues P, Domingues R, Martinho A, Botelho MF, Trindade H, Paiva A. Amniotic membrane extract differentially regulates human peripheral blood $\mathrm{T}$ cell subsets, monocyte subpopulations and myeloid dendritic cells. Cell Tissue Res 2018;373:459-476

28. Rossi D, Pianta S, Magatti M, Sedlmayr P, Parolini O. Characterization of the conditioned medium from amniotic membrane cells: prostaglandins as key effectors of its immunomodulatory activity. PLoS One 2012;7:e46956

29. Morandi F, Rizzo R, Fainardi E, Rouas-Freiss N, Pistoia $\mathrm{V}$. Recent advances in our understanding of HLA-G biology: lessons from a wide spectrum of human diseases. J Immunol Res 2016;2016:4326495

30. Sionov RV, Fridlender ZG, Granot Z. The multifaceted roles neutrophils play in the tumor microenvironment. Cancer Microenviron 2015;8:125-158

31. Shishikura K, Horiuchi T, Sakata N, Trinh DA, Shirakawa R, Kimura T, Asada Y, Horiuchi H. Prostaglandin E2 inhibits neutrophil extracellular trap formation through production of cyclic AMP. Br J Pharmacol 2016;173:319-331

32. Sabat R, Grütz G, Warszawska K, Kirsch S, Witte E, Wolk $\mathrm{K}$, Geginat J. Biology of interleukin-10. Cytokine Growth Factor Rev 2010;21:331-344

33. Loughman JA, Yarbrough ML, Tiemann KM, Hunstad DA. Local generation of kynurenines mediates inhibition of neutrophil chemotaxis by uropathogenic escherichia coli. Infect Immun 2016;84:1176-1183

34. Bak DH, Na J, Choi MJ, Lee BC, Oh CT, Kim JY, Han HJ, Kim MJ, Kim TH, Kim BJ. Anti-apoptotic effects of human placental hydrolysate against hepatocyte toxicity in vivo and in vitro. Int J Mol Med 2018;42:2569-2583

35. Schumacher A, Sharkey DJ, Robertson SA, Zenclussen AC. Immune cells at the fetomaternal interface: how the microenvironment modulates immune cells to foster fetal development. J Immunol 2018;201:325-334

36. Gupta A, Kedige SD, Jain K. Amnion and chorion membranes: potential stem cell reservoir with wide applications in periodontics. Int J Biomater 2015;2015:274082

37. Gomez-Lopez N, Romero R, Xu Y, Miller D, Leng Y, Panaitescu B, Silva P, Faro J, Alhousseini A, Gill N, Hassan SS, Hsu CD. The immunophenotype of amniotic fluid leukocytes in normal and complicated pregnancies. Am J Reprod Immunol 2018;79:e12827

38. Zeng MY, Miralda I, Armstrong CL, Uriarte SM, Bagaitkar J. The roles of NADPH oxidase in modulating neutrophil effector responses. Mol Oral Microbiol 2019;34:27-38

39. Gomez-Lopez N, Romero R, Garcia-Flores V, Xu Y, Leng Y, Alhousseini A, Hassan SS, Panaitescu B. Amniotic fluid neutrophils can phagocytize bacteria: a mechanism for mi- 
crobial killing in the amniotic cavity. Am J Reprod Immunol 2017;78:e12723

40. Gomez-Lopez N, Romero R, Xu Y, Leng Y, Garcia-Flores V, Miller D, Jacques SM, Hassan SS, Faro J, Alsamsam A,
Alhousseini A, Gomez-Roberts H, Panaitescu B, Yeo L, Maymon E. Are amniotic fluid neutrophils in women with intraamniotic infection and/or inflammation of fetal or maternal origin? Am J Obstet Gynecol 2017;217:693.e1-693.e16 